\title{
Comparison of T-Spot.TB and tuberculin skin test among silicotic patients
}

\author{
C.C. Leung*, W.C. Yam" ${ }^{\#}$, W.W. Yew", P.L. Ho\#, C.M. Tam, W.S. Law*, M.Y. Wong*, \\ M. Leung* and D. Tsui*
}

ABSTRACT: In the present study, T-Spot.TB and the tuberculin skin test (TST) were compared in the screening of latent tuberculosis infection among silicotic patients.

A conditional probability model was used to compare the potential clinical utilities of T-Spot.TB and TST performed on 134 silicotic subjects from December 1, 2004 to January 31, 2007. Data from a historical cohort were also reanalysed for further comparison.

Agreement with T-Spot.TB was best using a TST cut-off of $10 \mathrm{~mm}$. Age $\geqslant 65 \mathrm{yrs}$ independently predicted a tuberculin reaction $<10 \mathrm{~mm}$ (odds ratio $=3$ ), but not a negative T-Spot.TB response. Lower measures of agreement were observed among current smokers and those aged $\geqslant 65 \mathrm{yrs}$. Tuberculin reaction size was well correlated with both early secretary antigenic target 6 and culture filtrate protein 10 spot counts, except among current smokers. Within the current estimates of sensitivity (88-95\%) and specificity (86-99\%) for T-Spot.TB, the positive likelihood ratio for T-Spot.TB test would be substantially higher (6.29-95.0 versus $1.65-1.94)$ and negative likelihood ratio substantially lower $(0.05-0.14$ versus $0.32-0.41)$ than the corresponding ratios for the tuberculin test. A low tuberculosis risk differential was similarly observed between tuberculinnegative and untreated tuberculin-positive subjects in the historical cohort.

T-Spot.TB is likely to perform better than tuberculin test in the screening of latent tuberculosis infection among silicotic subjects.

KEYWORDS: Latent tuberculosis infection, silicosis, smoking

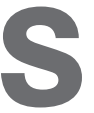
ilicotic subjects are at a high risk of developing tuberculosis (TB). Two previous studies in Hong Kong quantified the annual risk of TB in silicotic subjects, which is in the range of $\sim 3-5 \%$ per annum $[1,2]$. Tuberculin skin test (TST; tuberculin test) has been in regular use for the diagnosis of latent tuberculosis infection (LTBI) for many years. A positive reaction can result from infection by Mycobacterium tuberculosis, previous bacillus Calmette-Guérin (BCG) vaccination, or crossreaction caused by nontuberculous mycobacteria [3]. Most of the silicotic patients in the present study, with a mean age of 60 yrs, were born before a large-scale BCG vaccination was introduced in Hong Kong, but little data is available on the impact of other environmental mycobacteria. A cut-off point of $10 \mathrm{~mm}$ has regularly been employed to diagnose LTBI. The actual sensitivity is unknown because of the absence of a gold standard. Reduced sensitivity of tuberculin with age has been reported among residents of residential homes [4] and elderly patients with culture-confirmed TB [5]. Smoking, alcohol use and body mass index have been found to be independent predictors of a positive tuberculin reaction among silicotic subjects [6], but this could reflect either higher prevalence of LTBI or underlying differences in the immune status. Interferon (IFN)- $\gamma$ release blood assays have been introduced in recent years. Based on specific antigens identified through genomic research, they are less likely to be affected by previous BCG vaccination and infections with nontuberculous mycobacteria [7]. In the absence of a gold standard for LTBI, estimation of diagnostic sensitivity was primarily based on the testpositive rate among patients with culture-confirmed TB, and estimation of specificity on the test-negative rate among individuals with low risk of exposure in a low TB incidence area [3,8]. Preliminary evidence suggests a higher sensitivity and specificity of these tests than the traditional TST [8-11]. In the targeted screening for LTBI, comparison between these tests has mainly been performed using various measures of agreement, such as concordance/discordance or kappa measure, with or without correlation with
AFFILIATIONS

*Tuberculosis and Chest Service,

Centre for Health Protection, Dept of Health,

\#Dept of Microbiology and Centre of Infection, University of Hong Kong, and

"Tuberculosis and Chest Unit, Grantham Hospital, Hong Kong, China.

\section{CORRESPONDENCE}

\section{C.C. Leung}

Pneumoconiosis Clinic $4 / F$

8 Chai Wan Road

Shaukeiwan

Hong Kong

China

Fax: 8685229775940

E-mail: cc_leung@dh.gov.hk

Received:

May 072007

Accepted after revision:

October 122007

\section{SUPPORT STATEMENT}

This work is supported by research grants from the Pneumoconiosis

Compensation Fund Board and from the University of Hong Kong UDF Project-Research Centre of Emerging Infection Diseases (both Hong Kong, China).

\section{STATEMENT OF INTEREST}

None declared. 
exposure gradient [7-11]. However, the actual significance of such measures is difficult to interpret in day-to-day clinical practice, especially outside the contact settings. Therefore, the present authors conducted a study comparing an enzymelinked immunospot assay, T-Spot.TB (Oxford Immunotec Ltd, Abingdon, UK), with TST in the targeted screening of silicotic subjects, and employed a simple conditional model to examine how measurements of agreement could translate into clinically relevant performance characteristics in the target population.

\section{METHOD}

All confirmed silicotic patients with profusion of opacities at category 1 or above, without past history or current suspicion of active TB and not having been offered targeted screening and treatment of LTBI, were offered both TST and T-Spot.TB test when they attended the Pneumoconiosis Clinic (Hong Kong, China), the only compensation assessment centre for pneumoconiosis in Hong Kong. Background sociodemographic and clinical information was obtained together with checking old BCG scars and measurement of baseline body weight and height. After consent, TST was carried out by the Mantoux technique with two units of purified protein derivative-RT23 on one of the forearms, which was read after $48-72 \mathrm{~h}$. In addition, $8 \mathrm{~mL}$ of fresh blood was taken by venipuncture for T-Spot.TB, which was conducted and interpreted according to the supplier's protocol as detailed in the online supplementary data (E1).

The TST results, as defined by cut-off values of $\geqslant 5,10$ and $15 \mathrm{~mm}$, were compared in turn with the T-Spot.TB results for agreement. The effects of sociodemographic and clinical factors on the TST and T-Spot.TB test were also examined.

To facilitate the interpretation of comparison results, a simple deterministic model using conditional probabilities (supplementary data, E2) was employed to calculate the sensitivity, specificity, positive likelihood ratio, negative likelihood ratio [12], positive predictive value (PPV) and negative predictive value (NPV) of the T-Spot.TB test and TST as applied to the study cohort as follows. 1) Assuming the existing surrogate measures of sensitivity and specificity are valid, the following parameters for T-Spot.TB test (as based on a recently published meta-analysis [8]) were input into the model. Sensitivity (for adult population): $92 \%$ (95\% confidence interval (CI) $88-95 \%$ ); specificity: $92.5 \%$ (95\% CI $86-99 \%)$. 2) The positive and negative likelihood ratios of T-Spot.TB were calculated directly from the assumed sensitivity and specificity as previously described [12]. 3) The PPV and NPV of T-Spot.TB were calculated through the given assumptions of sensitivity and specificity and the distribution of positive and negative results among the test cohort by solving a set of simultaneous equations (supplementary data, E2). 4) The derived PPV and NPV of T-Spot.TB were then used as conditional probabilities for a true-positive or a true-negative for a positive and negative T-Spot.TB test, respectively, for the calculation of the sensitivity, specificity, positive likelihood ratio, negative likelihood ratio, PPV and NPV of the TST through a two-by-two table of these two tests. 5) The calculations were then repeated for sensitivity analysis, using upper and lower 95\% confidence limits of the current estimates of T-Spot.TB sensitivity and specificity.
The positive likelihood ratio reflects the relative likelihood of LTBI among test-positive subjects versus test-negative subjects [12]. In the presence of a low proportion (20-24\%) of TB cases, due to recent transmission in the local population $[13,14]$, it should indirectly reflect the relative risk of developing active TB for test-positive and test-negative subjects. Prospective data comparing the relative risk of active TB in test-positive with that in test-negative subjects are still pending for the new IFNrelease assays [8]. For the TST, a study involving a prospective follow-up of a cohort of 435 tuberculin-tested silicotic patients in the same location was recently published; details of the cohort have previously been described [6]. Largely because of patient refusal, a significant proportion of tuberculin-positive subjects were not treated for LTBI, thus allowing comparison of TB risk between tuberculin-negative and untreated tuberculin-positive subjects during an average follow-up of 5 yrs. After re-categorisation of active TB cases found in that cohort by baseline tuberculin and treatment status, the relative TB risks between different groups were compiled and compared with the predictions of the above model.

\section{Statistical analysis}

In univariate analysis, the Chi-squared test or Fisher exact test were used for unpaired categorical variables (across groups) as appropriate, and the McNemar test was used for paired proportions (within same subjects). For numerical variables, ANOVA, Mann-Whitney U-test and Wilcoxon signed ranks test were used as appropriate. Kappa measure and Spearman's rank correlation were used to assess agreement between tests and correlation of numerical test readings, respectively. Multiple logistic regression analysis was employed to control for confounding background/disease variables associated with the test outcome variables $(p<0.20)$ in univariate analysis. A two-tailed $\mathrm{p}$-value $<0.05$ was taken as statistically significant.

Approval for the study was obtained from the Ethics Committee of the Dept of Health of Hong Kong SAR. The TSpot.TB test kits were supplied at a reduced price by Oxford Immunotec Ltd.

\section{RESULTS}

From December 1, 2004 to January 31, 2007, 134 patients underwent both T-Spot.TB test and TST with an uptake rate of $\sim 50 \%$ among patients offered the tests during the course of clinic attendance. Only six $(4.3 \%)$ subjects showed an indeterminate T-Spot.TB test because of low cell counts after separation of the mononuclear cells. Repeat blood testing yielded a determinate result. There were no significant differences in age, presence of comorbidity and final result between those requiring and not requiring a repeat test (two tailed exact test, all $\mathrm{p}>0.05)$. The subject's background characteristics, as stratified by age ( $<65$ versus $\geqslant 65$ yrs), are summarised in table 1 . Only two subjects presented with a BCG scar; both were aged $<65$ yrs. One of the subjects showed a tuberculin reaction of $7 \mathrm{~mm}$ and a positive T-Spot.TB result. The other had a tuberculin reaction of $12 \mathrm{~mm}$ but a negative TSpot.TB result. There was no significant difference in the proportion of subjects with a tuberculin reaction $\geqslant 10 \mathrm{~mm}$ and a positive T-Spot.TB test (68.7 versus $64.2 \%$; McNemar test, $\mathrm{p}=0.391$ ). A higher proportion of subjects aged $<65$ yrs had a positive tuberculin reaction $(\geqslant 10 \mathrm{~mm})$ as compared with those 
aged $\geqslant 65$ yrs $(p=0.01)$, but no significant association was observed between T-Spot.TB test status and age (table 1).

Table 2 summarises the univariate analysis of the background characteristics according to TST and T-Spot.TB status. Older age was associated with a tuberculin reaction $<10 \mathrm{~mm}$, but no significant association was found between T-Spot.TB test and any of the characteristics listed in table 2. Higher proportions of current smokers than never- or ex-smokers were found to have a tuberculin reaction $\geqslant 10 \mathrm{~mm}(81.3$ versus $64.7 \% ; \mathrm{p}=0.078)$ and a positive T-Spot.TB test (78.1 versus $59.3 \%$; $\mathrm{p}=0.059)$, but the differences just failed to reach statistical significance. On multiple logistic regression analysis using predictor variables associated with test outcomes $(\mathrm{p}<0.20)$ in univariate analysis

\begin{tabular}{|c|c|c|c|c|}
\hline $\begin{array}{cc}\text { TABLE } 1 & \text { Distribl } \\
& \text { tubercl } \\
& \text { stratifie }\end{array}$ & $\begin{array}{l}\text { ution of b } \\
\text { ulin status } \\
\text { d by age }\end{array}$ & $\begin{array}{l}\text { ackground } \\
\text { and T-Spo }\end{array}$ & $\begin{array}{l}\text { characteristic } \\
\text { t.TB results }\end{array}$ & \\
\hline Variables & Overall & Age $<65$ yrs & Age $\geqslant 65$ yrs & p-value \\
\hline Subjects $n$ & 134 & 91 & 43 & \\
\hline Male sex & 97 & 98 & 95 & $0.59^{\#}$ \\
\hline Smoking status & & & & $0.33^{\circ}$ \\
\hline Never & 17.2 & 15.4 & 21 & \\
\hline Ex & 59 & 57 & 63 & \\
\hline Current & 24 & 28 & 16.3 & \\
\hline Regular alcohol use & 10.4 & 11.0 & 9.3 & $1.00^{\#}$ \\
\hline BCG scar & 1.5 & 2.2 & 0.0 & $1.00^{\#}$ \\
\hline With comorbidity $^{+}$ & 18.7 & 9.9 & 37 & $<0.001$ \\
\hline Principal job & & & & $<0.01^{\circ}$ \\
\hline Underground driller ${ }^{5}$ & 44 & 55 & 21 & \\
\hline Surface driller ${ }^{f}$ & 37 & 31 & 51 & \\
\hline Other & 18.7 & 14.3 & 28 & \\
\hline Nodule profusion & & & & $0.49^{\circ}$ \\
\hline Category 1 & 72 & 74 & 70 & \\
\hline Category 2 & 26 & 24 & 30 & \\
\hline Category 3 & 1.5 & 2.2 & 0 & \\
\hline Nodule size mm & & & & $0.94^{\circ}$ \\
\hline$<1.5$ & 26 & 25 & 28 & \\
\hline $1.5-3$ & 65 & 66 & 63 & \\
\hline $3-10$ & 9.0 & 8.8 & 9.3 & \\
\hline PMF & 19.4 & 16.5 & 26 & 0.21 \\
\hline $\begin{array}{l}\text { Tuberculin reaction } \\
\quad \geqslant 10 \mathrm{~mm}\end{array}$ & 69 & 77 & 51 & 0.01 \\
\hline Positive T-Spot.TB test & 64 & 68 & 56 & 0.17 \\
\hline Age yrs & $60.4 \pm 9.7$ & $54.8 \pm 5.3$ & $72.2 \pm 5.3$ & \\
\hline Cigarette pack-yrs & $21.4 \pm 20.3$ & $17.3 \pm 16.7$ & $30.1 \pm 24.4$ & $<0.001$ \\
\hline $\begin{array}{l}\text { Body mass index } \\
\mathrm{kg} \cdot \mathrm{m}^{-2}\end{array}$ & $24.2 \pm 3.0$ & $24.0 \pm 3.0$ & $24.5 \pm 3.2$ & 0.45 \\
\hline Dust exposure yr & $22.9 \pm 8.7$ & $20.4 \pm 7.2$ & $28.2 \pm 9.3$ & $<0.001$ \\
\hline
\end{tabular}

Data are presented as $\%$ or mean $\pm \mathrm{SD}$, unless otherwise stated. BCG: bacilli Calmette-Guérin; PMF: progressive massive fibrosis. * : Fisher exact, two-tailed p-value; ': univariate analysis of each variable by age group; ${ }^{+}$: diabetes mellitus, heart/cerebrovascular disease, malignancies, cirrhosis and renal and gastrointestinal diseases; hypertension alone was not included; ${ }^{\text {s. }}$ : caisson workers/tunnel workers/miners; $f$ : surface drillers/stone-splitters/drillers in construction trade or quarries. (table 2 ), age $\geqslant 65$ yrs remained an independent predictor of a negative tuberculin reaction $<10 \mathrm{~mm}$ (odds ratio (OR) 3, 95\% CI 1.38-6.55; $\mathrm{p}=0.006)$, but not a negative T-Spot.TB response (OR $1.57,95 \%$ CI 0.74-3.55; $\mathrm{p}=0.636)$, while current smoking did not significantly predict a positive tuberculin response $\geqslant 10 \mathrm{~mm}$ (OR 2.10, 95\% CI 0.77-5.82; $\mathrm{p}=0.146$ ) or a positive T-Spot.TB test (OR 2.27, 95\% CI 0.89-5.78; $\mathrm{p}=0.085$ ).

The median (interquartile range) tuberculin reaction sizes were $0 \quad(0.0-6.3) \mathrm{mm}$ and $16(14-20) \mathrm{mm}$ for subjects with a tuberculin reaction size $<10$ and $\geqslant 10 \mathrm{~mm}$, respectively. Of the 86 subjects with a positive T-Spot.TB test, 29 (33.7\%) had a net spot count (peptides well count-control well count) of six or more for the early secretary antigenic target (ESAT)6 peptides well alone, $18(20.9 \%)$ for the culture filtrate protein (CFP)10 peptides well alone, and 39 (45.3\%) for both. The median (interquartile range) net spot counts for the ESAT6 peptides well and CFP10 peptides well were 16.5 (6.0-32.0) and 10.5 (2.8-52.0), respectively (Wilcoxon signed ranks test, $\mathrm{p}=0.830$ ). Higher tuberculin reaction sizes (median 15.0 versus $10.0 \mathrm{~mm}$; Mann-Whitney U-test, $\mathrm{p}=0.001$ ), but not net ESAT6 well spot counts (median 6.0 versus 4.0; Mann-Whitney U-test, $\mathrm{p}=0.920$ ) or CFP10 well spot counts (median 3.0 versus 2.0; Mann-Whitney U-test, $\mathrm{p}=0.300$ ), were observed among subjects aged $<65$ yrs in comparison to those aged $\geqslant 65$ yrs. Higher tuberculin reaction sizes (median 16.0 versus $12.5 \mathrm{~mm}$; Mann-Whitney U-test, $\mathrm{p}=0.016$ ) and net ESAT6 well spot counts (median 15.5 versus 4.0; Mann-Whitney U-test, $\mathrm{p}=0.013$ ), but not CFP10 well spot counts (median 4.0 versus 3.0; Mann-Whitney U-test, $\mathrm{p}=0.243$ ), were observed among current smokers in comparison to among never- or ex-smokers. Table 3 summarises the correlation between tuberculin reaction size and the net spot counts for the ESAT6 and CFP10 peptide wells among the whole cohort before and after stratification by age and smoking.

Table 4 summarises the comparison of T-Spot.TB with the TST using different cut-off points. The concordance rates between T-Spot.TB and the TST were 71.6, 74.6 and $67.9 \%$ for cut-off points of 5, 10 and $15 \mathrm{~mm}$, respectively. Higher concordance rates were found among T-Spot.TB test-positive subjects than T-Spot.TB test-negative subjects for the tuberculin cut-off points of $5 \mathrm{~mm}(89.5$ versus $39.6 \%$; $<<0.001)$ and $10 \mathrm{~mm}(83.7$ versus $58.3 \% ; \mathrm{p}=0.001)$, but the opposite was true for the $15 \mathrm{~mm}$ cut-off ( 35.8 versus $64.2 \%$; $\mathrm{p}=0.037$ ). Overall, and for all subgroups (aged $<65$ yrs, aged $\geqslant 65$ yrs, current smokers, and never- and ex-smokers), the kappa measurement for agreement between T-Spot.TB and the TST was at a maximum with a cut-off reaction size of $10 \mathrm{~mm}$ for the TST. For all cut-offs, the kappa measurements of agreement between TST and TSpot.TB test were similar but greater for subjects aged $<65$ yrs than those aged $\geqslant 65$ yrs. Much lower kappa measurements were observed for current smokers in contrast with those for never- and ex-smokers.

Table 5 summarises the estimated sensitivity, specificity, positive and negative likelihood ratios, PPV and NPV of TSpot.TB and TST (cut-off $10 \mathrm{~mm}$ ) for the study cohort under the current estimates of T-Spot.TB sensitivity (88-95\% for adult population) and specificity (86-99\%) [8]. Substantial differences in test characteristics were observed between T-Spot.TB and TST across the full range of assumptions. The estimated 
TABLE 2 Distribution of background characteristics as stratified by tuberculin skin test and T-Spot.TB status

\begin{tabular}{|c|c|c|c|c|c|c|}
\hline \multirow[t]{2}{*}{ Variables } & \multicolumn{3}{|c|}{ Tuberculin reaction } & \multicolumn{3}{|c|}{ T-Spot.TB } \\
\hline & $<10 \mathrm{~mm}$ & $\geqslant 10 \mathrm{~mm}$ & $\mathrm{p}$-value & Negative & Positive & $p$-value \\
\hline Subjects $n$ & 45 & 88 & & 49 & 84 & \\
\hline Male sex & 98 & 97 & $1.00^{\#}$ & 100 & 95 & $0.30^{\#}$ \\
\hline Smoking status & & & $0.19^{\circ}$ & & & $0.14^{\circ}$ \\
\hline Current & 14.3 & 28 & & 14.6 & 29 & \\
\hline Alcohol use & 14.3 & 8.7 & 0.33 & 12.5 & 9.3 & 0.56 \\
\hline BCG scar & 2.4 & 1.1 & $0.53^{\#}$ & 2.1 & 1.2 & $1.00^{\#}$ \\
\hline Comorbidity $^{+}$ & 16.7 & 19.6 & 0.69 & 18.8 & 18.6 & 0.98 \\
\hline Principal job & & & $0.50^{\circ}$ & & & $0.31^{\star}$ \\
\hline Underground driller ${ }^{\S}$ & 38 & 47 & & 35 & 49 & \\
\hline Category 1 & 69 & 74 & & 73 & 72 & \\
\hline Category 2 & 29 & 25 & & 27 & 26 & \\
\hline Category 3 & 2.4 & 1.1 & & 0.0 & 2.3 & \\
\hline Nodule size mm & & & $0.88^{\circ}$ & & & $0.52^{\bullet}$ \\
\hline$<1.5$ & 26 & 26 & & 27 & 25 & \\
\hline $1.5-3$ & 67 & 64 & & 60 & 67 & \\
\hline $3-10$ & 7.1 & 9.8 & & 12.5 & 7.0 & \\
\hline PMF & 23.8 & 17.4 & 0.38 & 25 & 16.3 & 0.22 \\
\hline Age yrs & $63.6 \pm 9.0$ & $58.9 \pm 9.7$ & 0.01 & $61.3 \pm 9.8$ & $59.9 \pm 9.7$ & 0.42 \\
\hline Cigarette pack-yr & $24.7 \pm 24.6$ & $19.9 \pm 18.0$ & 0.20 & $24.8 \pm 20.2$ & $19.5 \pm 18.8$ & 0.28 \\
\hline Body mass index $\mathrm{kg} \cdot \mathrm{m}^{-2}$ & $23.8 \pm 3.5$ & 24. $3 \pm 2.8$ & 0.35 & $24.0 \pm 3.5$ & $24.4 \pm 2.7$ & 0.45 \\
\hline Dust exposure yr & $23.2 \pm 9.7$ & $22.8 \pm 8.3$ & 0.77 & $23.8 \pm 7.6$ & $22.4 \pm 9.3$ & 0.39 \\
\hline
\end{tabular}

TABLE 3 Correlation between tuberculin reaction size and the net-spot counts in T-Spot.TB test among the whole cohort before and after stratification by age and smoking

\begin{tabular}{|c|c|c|c|c|c|}
\hline \multirow[t]{2}{*}{ Group } & \multirow[t]{2}{*}{ Subjects $\mathrm{n}$} & \multicolumn{2}{|c|}{ ESAT6 well } & \multicolumn{2}{|c|}{ CFP10 well } \\
\hline & & Correlation ${ }^{\#}$ & $\mathrm{p}$-value & Correlation ${ }^{\#}$ & $p$-value \\
\hline $\begin{array}{l}\text { Whole } \\
\text { cohort }\end{array}$ & 134 & 0.336 & $<0.001$ & 0.243 & $<0.01$ \\
\hline Age $<65$ yrs & 91 & 0.324 & $<0.01$ & 0.253 & 0.02 \\
\hline Age $\geqslant 65 \mathrm{yrs}$ & 43 & 0.422 & $<0.01$ & 0.197 & 0.21 \\
\hline $\begin{array}{l}\text { Current } \\
\text { smokers }\end{array}$ & 32 & 0.030 & 0.87 & -0.096 & 0.60 \\
\hline $\begin{array}{c}\text { Never- or ex- } \\
\text { smokers }\end{array}$ & 102 & 0.375 & $<0.001$ & 0.308 & $<0.001$ \\
\hline
\end{tabular}

ESAT: early secretary antigenic target; CFP: culture filtrate protein. \#. Spearman's rank correlation coefficients between the tuberculin reaction size and the net spot count (test well count-negative control well count) of T-Spot.TB test. sensitivity of TST varied from $78.7-81.6 \%$ and specificity from $52.4-57.9 \%$. The positive likelihood ratio for T-Spot.TB test was higher (6.29-95.0 versus 1.65-1.94) and negative likelihood ratio lower $(0.05-0.14$ versus $0.32-0.41)$ than the corresponding ratios of the TST.

From a separate historical cohort of 435 silicotic patients who were tuberculin tested between August 1, 1995 and December 31,2002 [5], $17(14.4 \%)$ out of 118 patients with a TST $<10 \mathrm{~mm}$, $45(20.8 \%)$ out of 216 untreated patients with a TST $\geqslant 10 \mathrm{~mm}$, and $11(10.9 \%)$ out of 101 patients with a TST $\geqslant 10 \mathrm{~mm}$ and treated for LTBI (either 6 months of isoniazid or 2 months of rifampicin and pyrazinamide at a treatment completion rate of $70 \%$ ) developed active TB after $5.2 \pm 2.3$ yrs of follow-up until the end of 2005. The corresponding TB incidence rates were 2,596 (tuberculin-negative subjects), 3,778 (untreated tuberculinpositive subjects) and 2,582 (treated tuberculin-positive subjects) per 100,000 person-yrs, respectively. The TB incidence ratios were observed to be $0.69(2,596$ out of 3,778$)$ between tuberculinnegative and untreated tuberculin-positive subjects, and 0.68 $(2,582$ out of 3,778$)$ between treated and untreated tuberculinpositive subjects. The relatively low risk differential between tuberculin-negative and untreated tuberculin-positive subjects 


\begin{tabular}{|c|c|c|c|c|c|c|}
\hline \multirow{3}{*}{$\begin{array}{l}\text { TABLE } 4 \\
\text { Tuberculin } \\
\text { test }\end{array}$} & \multicolumn{6}{|c|}{$\begin{array}{l}\text { Comparison of T-Spot.TB with tuberculin test } \\
\text { using different cut-off points }\end{array}$} \\
\hline & \multicolumn{3}{|c|}{ T-Spot.TB } & \multirow[t]{2}{*}{ Concordance } & \multirow[t]{2}{*}{ Kappa } & \multirow[t]{2}{*}{ p-value } \\
\hline & All & Negative & Positive & & & \\
\hline \multicolumn{7}{|c|}{ Whole cohort } \\
\hline$<5 \mathrm{~mm}$ & $28(100)$ & 19 (67.9) & $9(32.1)$ & & & \\
\hline$\geqslant 5 \mathrm{~mm}$ & $\begin{array}{c}106 \\
(100)\end{array}$ & $29(27.4)$ & 77 (72.6) & 71.6 & 0.321 & $<0.001$ \\
\hline$<10 \mathrm{~mm}$ & $42(100)$ & $28(66.7)$ & 14 (33.3) & & & \\
\hline$\geqslant 10 \mathrm{~mm}$ & $92(100)$ & $20(21.7)$ & $72(78.3)$ & 74.6 & 0.432 & $<0.001$ \\
\hline$<15 \mathrm{~mm}$ & $71(100)$ & 38 (53.5) & $33(46.5)$ & & & \\
\hline$\geqslant 15 \mathrm{~mm}$ & $63(100)$ & $10(15.9)$ & $53(84.1)$ & 67.9 & 0.369 & $<0.001$ \\
\hline \multicolumn{7}{|l|}{ Age $<65$ yrs } \\
\hline Overall & $91(100)$ & 29 (31.9) & $62(68.1)$ & & & \\
\hline$\geqslant 5 \mathrm{~mm}$ & $78(100)$ & $19(24.4)$ & 59 (75.6) & 75.8 & 0.347 & $<0.001$ \\
\hline$\geqslant 10 \mathrm{~mm}$ & $70(100)$ & $14(20.0)$ & $56(80.0)$ & 78.0 & 0.454 & $<0.001$ \\
\hline$\geqslant 15 \mathrm{~mm}$ & $52(100)$ & $8(15.4)$ & $44(84.6)$ & 71.4 & 0.397 & $<0.001$ \\
\hline \multicolumn{7}{|c|}{ Age $\geqslant 65$ yrs } \\
\hline Overall & $43(100)$ & $19(44.2)$ & $24(55.8)$ & & & \\
\hline$\geqslant 5 \mathrm{~mm}$ & $28(100)$ & $10(35.7)$ & $18(64.3)$ & 62.8 & 0.229 & 0.13 \\
\hline$\geqslant 10 \mathrm{~mm}$ & $22(100)$ & $6(27.3)$ & $16(72.7)$ & 67.4 & 0.347 & 0.02 \\
\hline$\geqslant 15 \mathrm{~mm}$ & $11(100)$ & $2(18.2)$ & $9(81.8)$ & 60.5 & 0.252 & 0.04 \\
\hline \multicolumn{7}{|l|}{$\begin{array}{l}\text { Current } \\
\text { smokers }\end{array}$} \\
\hline Overall & $32(100)$ & 7 (21.9) & $25(78.1)$ & & & \\
\hline$\geqslant 5 \mathrm{~mm}$ & $29(100)$ & $7(24.1)$ & $22(75.9)$ & 68.8 & -0.151 & 0.34 \\
\hline$\geqslant 10 \mathrm{~mm}$ & $26(100)$ & 5 (19.2) & $21(80.8)$ & 71.9 & 0.133 & 0.45 \\
\hline$\geqslant 15 \mathrm{~mm}$ & $22(100)$ & $4(18.2)$ & $18(81.8)$ & 65.6 & 0.129 & 0.45 \\
\hline \multicolumn{7}{|c|}{$\begin{array}{l}\text { Never- and } \\
\text { ex-smokers }\end{array}$} \\
\hline Overall & $\begin{array}{c}102 \\
(100)\end{array}$ & 41 (40.2) & $61(59.8)$ & & & \\
\hline$\geqslant 5 \mathrm{~mm}$ & $77(100)$ & 22 (28.6) & 55 (71.4) & 72.5 & 0.390 & $<0.001$ \\
\hline$\geqslant 10 \mathrm{~mm}$ & $66(100)$ & 15 (22.7) & $51(77.3)$ & 75.5 & 0.480 & $<0.001$ \\
\hline$\geqslant 15 \mathrm{~mm}$ & $41(100)$ & $6(14.6)$ & 35 (85.4) & 68.6 & 0.396 & $<0.001$ \\
\hline
\end{tabular}

Data are presented as \% (\% by rows).

was generally in line with the low positive likelihood ratios of TST as estimated from the conditional probability model (table 5).

\section{DISCUSSION}

In the present study, moderate agreement was found between T-Spot.TB and the TST using $10 \mathrm{~mm}$ as a cut-off point among a high-risk cohort of silicotic subjects in Hong Kong (table 4). However, older age was significantly associated with a negative TST response while no such associations were observed for T-Spot.TB (table 2). There was also a trend towards association between current smokers and a positive response in both tests. Poorer agreement was observed between T-Spot.TB and TST among older subjects and current smokers (table 4). Dissociation of T-Spot.TB spot counts from the TST size was also observed among current smokers (table 3). Within the current estimates of its sensitivity and specificity [8], the T-Spot.TB test would be expected to perform significantly better than the TST (table 5).

A lower sensitivity of TST among elderly subjects is well reported in previous studies $[4,5,15]$. In the current study, the lower percentage of tuberculin-positive subjects among those aged $\geqslant 65$ yrs probably reflected lower test sensitivity, rather than lower prevalence of LTBI. Among this fully ambulant cohort, no significant difference in comorbidities (other than silicosis and hypertension) was observed between tuberculinpositive and tuberculin-negative subjects (table 2), despite the higher prevalence of comorbidities among the elderly. Therefore, age appeared to affect the interpretation of TST even in the absence of major comorbidities. Conversely, TSpot.TB was not significantly affected by age, and this could support its role in the targeted screening of LTBI among elderly subjects.

The association between smoking and a positive tuberculin reaction is also well reported in the literature $[6,16,17]$. However, in contrast to the effect of age, it is usually assumed to indicate a higher LTBI prevalence, likely to be as a result of increased exposure. In the present study, significantly higher tuberculin reaction sizes and net ESAT6 well counts were observed among current smokers, in contrast with never- and ex-smokers. It is noteworthy that the association of smoking and tuberculin-positivity, as observed in the present and an earlier silicotic cohort [6], was seen predominantly among current smokers, with ex-smokers at a much lower risk. The total number of cigarette pack-yrs did not increase the risk of a positive response (table 2). The hypothesis of increased exposure among smokers (and hence higher true prevalence of LTBI) might not be adequate to account for such observations. As smoking has been well associated with inflammatory responses within the lungs [18-20], there remains a possibility that it could boost reactivity to tuberculin and the more specific antigens. The dissociation of the tuberculin reaction size from the T-Spot.TB spot counts among current smokers could also suggest that the magnitude of such boosting differs for different antigens.

The agreement between T-Spot.TB and TST was at a maximum using a cut-off of $10 \mathrm{~mm}$ (table 4), supporting previous use of such a cut-off among silicotic subjects, despite the recommendation of a lower cut-off of $5 \mathrm{~mm}$ by some authorities [3]. However, the agreement between the T-Spot.TB test and TST of $75.9 \% \quad(\mathrm{kappa}=0.474)$ was somewhat lower than that previously reported for BCG unvaccinated immunocompetent subjects in a previous study [11]. A higher mean age is likely to have contributed to such an observation, in line with the lower agreement observed among subjects aged $\geqslant 65 \mathrm{yrs}$.

As shown in table 5, under the deterministic conditional model and the ranges of T-Spot.TB sensitivity and specificity derived from a recent meta-analysis, the TST would have a sensitivity of $78.7-81.6 \%$, which was in reasonable agreement with the estimated tuberculin sensitivity (68-78\% for adult population) in the same meta-analysis [8]. However, the specificity of 52.4$57.9 \%$ was exceptionally low for a group with very low BCG vaccination coverage [8]. Cross-reactivity from previous exposure to nontuberculous mycobacteria could be a possible explanation [3], but differing abilities between T-Spot.TB and 


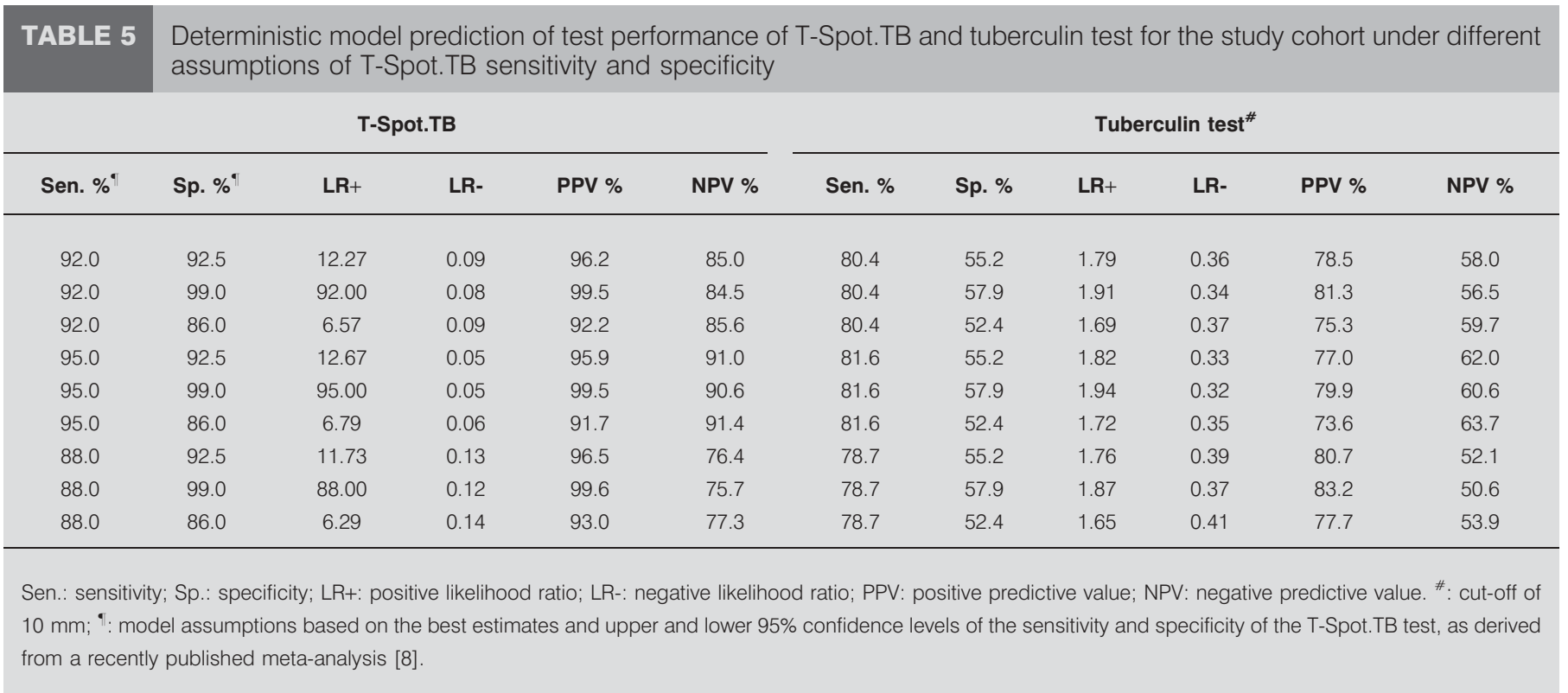

TST in detecting recent and remote infection might be an alternative explanation [21, 22]. Overall, a very low discriminating power of the TST was predicted, with a positive likelihood ratio consistently $<2$ and a negative likelihood ratio of $\sim 0.33$ (table 5 ). A relative risk of 0.69 was actually observed between tuberculin-negative and untreated tuberculin-positive subjects in a similar historical cohort. The residual risk among those screened as negative was $>2,500$ out of 100,000 personyrs. New infection or reinfection after tuberculin testing cannot account for the high residual risk, as previous molecular analysis of culture isolates showed only a low percentage of clustering, suggesting a low proportion of disease (20-24\%) due to recent transmission in Hong Kong [13, 14].

Silicotic patients in high TB prevalence areas are a well-known high-risk group for targeted screening of LTBI. Although the study was based on a convenient sample of silicotic patients attending a compensation assessment centre, this is the usual clinical setting where such targeted screening is offered. The absence of a gold standard remains a major problem in the comparison of diagnostic tests for LTBI. Similar to other studies [7-11, 21-24], no direct information was provided as to how well the new IFN- $\gamma$ release assay was able to predict subsequent risk of active TB disease. Indirect estimates of sensitivity and specificity through surrogate measures might not reflect actual test performance for the latent infection state. Although the specific antigens employed in the new test might help to reduce interference by cross-reactivity, insufficient information is available regarding the ability to differentiate between recent and remote infections. However, the derived sensitivity of TST was in keeping with previous estimates from surrogate measures. The limited discriminating power of the TST under the model was also supported by actual observation in a similar historical cohort in the same location, even though it must be admitted that the two cohorts were not identical [6].

Notwithstanding all the potential limitations, the present study highlighted potentially major differences in performance between the T-Spot.TB test and the tuberculin skin test among silicotic patients in a high tuberculosis prevalence setting. Further studies are needed to identify an optimal strategy for targeted screening of latent tuberculosis infection in such situations.

\section{REFERENCES}

1 A double-blind placebo-controlled clinical trial of three antituberculosis chemoprophylaxis regimens in patients with silicosis in Hong Kong, Hong Kong Chest Service/ Tuberculosis Research Centre, Madras/British Medical Research Council. Am Rev Respir Dis 1992; 145: 36-41.

2 Chang KC, Leung CC, Tam CM. Tuberculosis risk factors in a silicotic cohort in Hong Kong. Int J Tuberc Lung Dis 2001; 5: 177-184.

3 American Thoracic Society. Targeted tuberculin testing and treatment of latent tuberculosis infection. MMWR Recomm Rep 2000; 49: 1-51.

4 Nisar M, Williams CS, Ashby D, Davies PD. Tuberculin testing in residential homes for the elderly. Thorax 1993; 48: 1257-1260.

5 Leung CC, Yew WW, Chan CK, et al. Tuberculosis in older people: a retrospective and comparative study from Hong Kong. J Am Geriatr Soc 2002; 50: 1219-1226.

6 Leung CC, Yew WW, Law WS, et al. Smoking and tuberculosis among silicotic patients. Eur Respir J 2007; 29: 745-750.

7 Richeldi L. An update on the diagnosis of tuberculosis infection. Am J Respir Crit Care Med 2006; 174: 736-742.

8 Menzies D, Pai M, Comstock G. Meta-analysis: new tests for the diagnosis of latent tuberculosis infection: areas of uncertainty and recommendations for research. Ann Intern Med 2007; 146: 340-354.

9 Lalvani A, Pathan AA, Durkan H, et al. Enhanced contact tracing and spatial tracking of Mycobacterium tuberculosis infection by enumeration of antigen-specific $\mathrm{T}$ cells. Lancet 2001; 23: 2017-2021.

10 Ewer K, Deeks J, Alvarez L, et al. Comparison of T-cellbased assay with tuberculin skin test for diagnosis of 
Mycobacterium tuberculosis infection in a school tuberculosis outbreak. Lancet 2003; 361: 1168-1173.

11 Ferrara G, Losi M, D'Amico R, et al. Use in routine clinical practice of two commercial blood tests for diagnosis of infection with Mycobacterium tuberculosis: a prospective study. Lancet 2006; 367: 1328-1334.

12 Grimes DA, Schulz KF. Refining clinical diagnosis with likelihood ratios. Lancet 2005; 365: 1500-1505.

13 Chan-Yeung M, Tam CM, Wong H, et al. Molecular and conventional epidemiology of tuberculosis in Hong Kong: a population-based prospective study. J Clin Microbiol 2003; 41: 2706-2708.

14 Chan-Yeung M, Kam KM, Leung CC, et al. Populationbased prospective molecular and conventional epidemiological study of tuberculosis in Hong Kong. Respirology 2006; 11: 442-448.

15 Stead WW, To T. The significance of the tuberculin skin test in elderly persons. Ann Intern Med 1987; 107: 837-842.

16 Anderson RH, Sy FS, Thompson S, Addy C. Cigarette smoking and tuberculin skin test conversion among incarcerated adults. Am J Prev Med 1997; 13: 175-179.

17 Plant AJ, Watkins RE, Gushulak B, et al. Predictors of tuberculin reactivity among prospective Vietnamese migrants: the effect of smoking. Epidemiol Infect 2002; 128: 37-45.

18 Bracke KR, D’hulst AI, Maes T, et al. Cigarette smokeinduced pulmonary inflammation and emphysema are attenuated in CCR6-deficient mice. J Immunol 2006; 177: 4350-4359.

19 Reynolds PR, Cosio MG, Hoidal JR. Cigarette smokeinduced Egr-1 upregulates proinflammatory cytokines in pulmonary epithelial cells. Am J Respir Cell Mol Biol 2006; 35: 314-319.

20 Szulakowski P, Crowther AJ, Jimenez LA, et al. The effect of smoking on the transcriptional regulation of lung inflammation in patients with chronic obstructive pulmonary disease. Am J Respir Crit Care Med 2006; 174: 41-50.

21 Arend SM, Thijsen SF, Leyten EM, et al. Comparison of two interferon- $\gamma$ assays and tuberculin skin test for tracing tuberculosis contacts. Am J Respir Crit Care Med 2007; 175: 618-627.

22 Leyten EM, Arend SM, Prins C, Cobelens FG, Ottenhoff TH, van Dissel JT. Discrepancy between Mycobacterium tuberculosis-specific $\gamma$ interferon release assays using short and prolonged in vitro incubation. Clin Vaccine Immunol 2007; 14: 880-885.

23 Kang YA, Lee HW, Yoon HI, et al. Discrepancy between the tuberculin skin test and the whole-blood interferon- $\gamma$ assay for the diagnosis of latent tuberculosis infection in an intermediate tuberculosis-burden country. JAMA 2005; 293: 2756-2761.

24 Pai M, Gokhale K, Joshi R, et al. Mycobacterium tuberculosis infection in health care workers in rural India: comparison of a whole-blood interferon- $\gamma$ assay with tuberculin skin testing. JAMA 2005; 293: 2746-2755. 